\title{
Advanced Performance Metrics and their Application to the Sensitivity Analysis for Model Validation and Calibration
}

\author{
Urmila Agrawal, Member, IEEE, Pavel Etingov, Member, IEEE, and Renke Huang, Member, IEEE
}

\begin{abstract}
High-quality generator dynamic models are critical to reliable and accurate power systems studies and planning. With the availability of PMUs, measurement-based approach for model validation has gained significant prominence. In this approach, the quality of a model is analyzed by visually comparing measured generator response with the model-based simulated response for large system disturbances. This paper proposes a new set of performance metrics to assess the model validation results to facilitate automation of the model validation process. In the proposed methodology, first, the slow governor response and comparatively faster oscillatory response are separated, and then a separate set of performance metrics is calculated for each of these two components. These proposed metrics quantify the mismatch between the actual and model-based response in a comprehensive manner without missing any information enabling automation of the process. Furthermore, in this paper, we are also proposing that the sensitivity analysis for model calibration be performed with respect to the proposed metrics for the systematic identification of key parameters. Results obtained using both simulated and real-world case-studies validate the effectiveness of the proposed performance metrics for model validation and their application to the sensitivity analysis for model calibration.
\end{abstract}

Index Terms-Model validation and calibration, performance metrics, oscillatory response, governor response, signal Processing, PMU measurements, sensitivity analysis.

\section{INTRODUCTION}

$\mathbf{H}$ IGH quality dynamic model of generators are critical to reliable and economical power system operations and planning. Dynamic studies for various system disturbances, such as faults, generation loss, line trip, etc., is carried out using these models for both short and long term planning. These studies provide information on several aspects of power systems dynamic stability such as rotor angle stability, damping ratio of system modes, primary frequency response, system frequency and voltage recovery, etc., and identify contingencies that can result in system instability and stability constrained transmission paths. The accuracy of these studies heavily depends on the quality of dynamic models used, thereby making validation and calibration of generator dynamic models critically important. The need for accurate and up-to-date dynamic models for reliable and economical grid operations and planning was reinforced after the well-known

This work is supported by the U.S. Department of Energy's Office of Electricity through through the consortium for electric reliability technology solutions (CERTS) and Technology Commercialization Fund (TCF) programs. Pacific Northwest National Laboratory is operated by Battelle for DOE under contract DE-AC05-76RL01830.

U. Agrawal, P. Etingov and R. Huang are with the Pacific Northwest National Laboratory, Richland, WA, 99352 USA E-mail: urmila.agrawal@pnnl.gov
1996 western grid blackout. The planning Western System Coordinating Council (WSCC) model could not replicate the unstable system oscillations observed following the series of events that led to the system-wide outage [1]. NERC has since then required all generators having capacity of greater than 10 MVA to be validated every five years. Also, Reliability Standards MOD-026 [2] and MOD-027 [3] were developed to provide guidelines for generator model validation.

Traditional methods for validating generator dynamic models include staged and standstill frequency response testing [4]. These methods involve physical testing of the generators and therefore generators to be validated remain unavailable for normal operations. Even though these methods provide high quality dynamic models, these methods are technically difficult and are expensive [4]. With the availability of Phasor Measurement Units (PMUs), measurement-based validation methods have become widely-accepted [5]-[11]. This method requires PMUs to be installed at the point of interconnection (POI) of each generator to be validated. The PMU measurements recorded at these locations for a grid disturbance are then used as play-in signals to validate generator dynamic models as shown in Fig. 1 [12]. This approach of validating dynamic models is available in several power systems simulator such as GE PSLF, SIEMENS PTI PSSE, PowerWorld Simulator and TSAT [13].

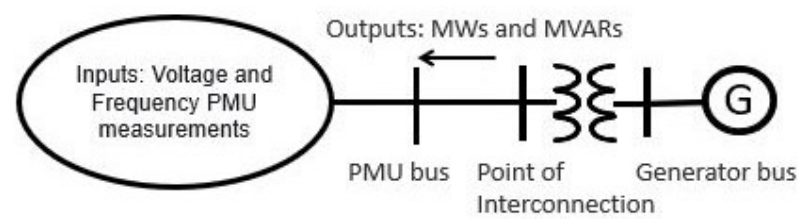

Fig. 1. Generator dynamic model validation using play-in PMU measurements

Several tools have been developed to validate generator dynamic modele using play-in PMU measurements, such as power plant model validation (PPMV) tool by Bonneville Power Administration (BPA) and Pacific Northwest National Laboratory (PNNL) [13], generator parameter validation (GPV) tool by Electric Power Group [14] and Power Plant Model Verification (PPMVer) tool by ISO-New England [7]. Current practices of analyzing model validation results include visually comparing actual generator real and reactive power response measured using PMUs with the simulated model-based response corresponding to a large system event [7], [15]. Some examples of metrics that have been proposed for quantifying mismatch between actual and simulated generator response include root-mean square error (RMSE) used in [13], peak 
value and peak-time of the first swing and steady-state error used in [16], normalized RMSE metric proposed in [15], [17]. Similar to the RMSE metric, the optimization function used for for model calibration in [18] defines waveform similarity metric that quantifies the mismatch between actual and modelbased response based on similarity of the curves. One other proposed metric include magnitude-shape similarity metric, which is based on frequency domain, first defined in [19] and later applied for quantifying model validation results in [20], [21]. This metric finds a weighted average of the similarity measure of the magnitude and phase spectra in frequency domain. All these metrics can help analyze model validation results and provide information on the quality of the model. However, the information provided by these metrics is limited and can only tell if the model is good or not. If the model validation process is to be automated using these metrics, critical information on mismatch between specific aspects of dynamic response could be lost which would otherwise have been obvious from a simple visual inspection. This information can specifically be useful for identification of key model parameters for model calibration.

Therefore, in this paper, we are proposing a new set of metrics for quantifying mismatch between the actual and model-based response that takes into consideration mismatch between specific aspects of generator dynamic response. In the proposed methodology, first the slow governor and fast oscillatory response are separated using a low-pass filter and then separate set of metrics are calculated for each of these two components of generator dynamic response; phase and magnitude similarity metric for generator oscillatory response [22], and delay, peak value, peak-value time, rise-time and steady-state value for generator governor response. These new proposed metrics are simple, intuitive and yet comprehensive in nature and can easily provide information on specific aspect of dynamic response that has mismatch. For example, in the 2nd case-study example used in this paper, which is based on real-world data, the mismatch was mostly in the governor response time (given by proposed delay metric). A simple RMSE metric could not have provided this information, however, the proposed metrics can provide this information without having to visually analyze the results, and thereby facilitating the automation of model validation process. Furthermore, proposed metrics can help with the identification of key model parameters that need to be tuned for model calibration. In several references, such as [23]-[25], the key parameters are identified by performing sensitivity analysis with respect to the RMSE metric that quantifies the change in the model-based response caused by a change in selected model parameter. There can be several parameters that are sensitive to the RMSE metric that may or may not be needed to be tuned at all. For example, if the model mismatch is only in the governor response, then performing sensitivity analysis with respect to the RMSE metric will select all parameters irrespective of the type of impact on model response, which includes both oscillatory and governor. For this purpose, in this paper, we are proposing that the sensitivity analysis be performed with respect to the proposed metrics. This approach can help identify parameters which are sensitive to specific aspect of generator response that has mismatch between actual and model-based response and thereby help with the identification of the key parameters in a more systematic and scientific manner as compared to the current practices. These parameters then can be used for model calibration using existing model calibration methodology, such as advanced ensemble Kalman filter method [25]. To summarize, the main contributions of the paper are the new set of performance metrics to judge the quality of the models and their application to the sensitivity analysis to identify problematic parameters that need to be calibrated, and facilitating the automation of model validation process without losing any information that could have been obtained by visual inspection.

The rest of the paper is organized as follows: Section II discusses background theory required to develop the proposed methodology, Section III presents a detailed description of the proposed methodology, Section IV provides results and discussion for proposed metrics and their application to the sensitivity analysis, and Section V concludes the paper.

\section{BACKGROUND THEORY}

This section briefly describes the Prony method used for quantifying mismatch for generator oscillatory response, and characteristics of the step-response of a system used for quantifying mismatch for generator governor response.

\section{A. Prony method}

While any modal analysis method, such as Prony [26] and Matrix-pencil [27], can be used to obtain the metrics for generator oscillatory response, Prony analysis method is used in this paper to illustrate the methodology of the proposed metrics for oscillatory response. The Prony method consists of three steps as described in [26]. Let the $N$ samples of measurements be given by $y[0], y[1], \ldots, y[N-1]$.

1) In the first step, a discrete linear prediction model (LPM) is obtained, that fits the signal, by solving a linear leastsquares problem given by

$$
\mathbf{Y} \boldsymbol{a}=\mathbf{y}
$$

where

$$
\begin{gathered}
\boldsymbol{a}=\left[\begin{array}{llll}
a_{1} & a_{2} & \cdots & a_{n}
\end{array}\right]^{T} \\
\mathbf{y}=\left[\begin{array}{llll}
y[n+0] & y[n+1] & \cdots & y[N-1
\end{array}\right]^{T} \\
\mathbf{Y}=\left[\begin{array}{cccc}
y[n-1] & y[n-2] & \cdots & y(0) \\
y[n-0] & y[n-1] & \cdots & y(1) \\
\vdots & \cdots & \cdots & \vdots \\
y[N-2] & y[N-3] & \cdots & y(N-n-1)
\end{array}\right] \text { and }
\end{gathered}
$$

$n$ is the model order selected to obtain system mode estimates. The least squares solution of (1) is given by

$$
\hat{\boldsymbol{a}}=\mathbf{Y}^{\dagger} \mathbf{y},
$$

where $\dagger$ denoted pseudo-inverse of a matrix. The n-th order polynomial equation is then given by

$$
1+a_{1} z^{-1}+a_{2} z^{-2}+\ldots+a_{n} z^{-n}=0
$$


2) In the second step, mode estimates, given by $\hat{\lambda}_{i}=\omega_{i}+$ $j \sigma_{i}$ for $i=1$ to $n$, are calculated using

$$
\hat{\lambda}_{i}=\frac{1}{\Delta T} \log \hat{z}_{i},
$$

where $\left\{\hat{z}_{i}\right\}_{i=1}^{n_{a}}$ are the roots of the estimated $\mathrm{n}$-th order polynomial equation given by (3), $\Delta T$ is the sampling time period of the measurements, $\omega_{i}$ is the frequency of each mode in $\mathrm{rad} / \mathrm{sec}$, and $\sigma_{i}$ is the damping coefficient. The damping ratio of each $i^{t h}$ mode is given by

$$
\hat{\zeta}_{i}=\frac{-\sigma_{i}}{\sqrt{\omega_{i}^{2}+\sigma_{i}^{2}}} .
$$

3) In the final step, the initial amplitude and phase of each mode, given by the phasor estimate $\hat{B}_{i}$, is calculated solving

$$
\mathbf{Z B}=\mathbf{y}^{\prime},
$$

where

$$
\begin{gathered}
\mathbf{Z}=\left[\begin{array}{cccc}
z_{1}^{0} & z_{2}^{0} & \cdots & z_{n}^{0} \\
z_{1}^{1} & z_{2}^{1} & \cdots & z_{n} 1 \\
\vdots & \cdots & \cdots & \vdots \\
z_{1}^{N-1} & z_{2}^{N-1} & \cdots & z_{n}^{N-1}
\end{array}\right] \\
\boldsymbol{B}=\left[\begin{array}{llll}
B_{1} & B_{2} & \cdots & B_{n}
\end{array}\right]^{T} \text { and } \\
\mathbf{y}^{\prime}=\left[\begin{array}{llll}
y[0] & y[1] & \cdots & y[N-1
\end{array}\right]^{T} .
\end{gathered}
$$

The least squares solution of (6) is given by

$$
\hat{B}=\mathbf{Z}^{\dagger} \mathbf{y}^{\prime},
$$

Following steps 1 to 3, mode estimates are given by (4) and mode shape of each mode is given by (7).

\section{Validation of mode estimates:}

Only estimating modes is not sufficient unless the mode estimates are validated [28]. For validating mode estimates, the original signal is compared with the reconstructed signal given by

$$
\hat{y}[k]=\sum_{i=1}^{n_{r}+n_{c}} \hat{y}_{i}[k],
$$

where $n_{r}$ is the number of real modes, $n_{c}$ is the number of pair of complex modes and $\hat{y}_{i}[k]$ is the contribution of the $i^{t h}$ mode to the signal given by

$$
\begin{aligned}
\hat{y}_{i}[k] & =\hat{B}_{i} \hat{z}_{i}^{k} \quad \text { for real mode } \\
& =2 * \Re\left(\hat{B}_{i} \hat{z}_{i}^{k}\right) \quad \text { for complex mode pair. }
\end{aligned}
$$

The goodness of fit metric is calculated using [26]

$$
G o F=1-\frac{\|y[k]-\hat{y}[k]\|}{\|y[k]\|},
$$

where $\|$.$\| denotes root-mean-square norm. Using (10), model$ order $n$ is selected that gives the best fit between the original and reconstructed signal.

\section{Sorting of system mode estimates}

As described in [28], not all mode estimates represent actual modes. Some of the mode estimates are spurious ones and need to be discarded. One of the ways of distinguishing actual mode estimates and spurious ones is to rank mode estimates based on their energy given by

$$
E_{i}=\sum_{k=1}^{N} \hat{y}_{i}[k]^{2}
$$

The modes having insignificantly small energy as compared to the highest energy can be discarded.

\section{B. Characteristics of the step-response of a system}

Governor response of a generator can be represented by the step-response of a system as shown in Fig. 2.

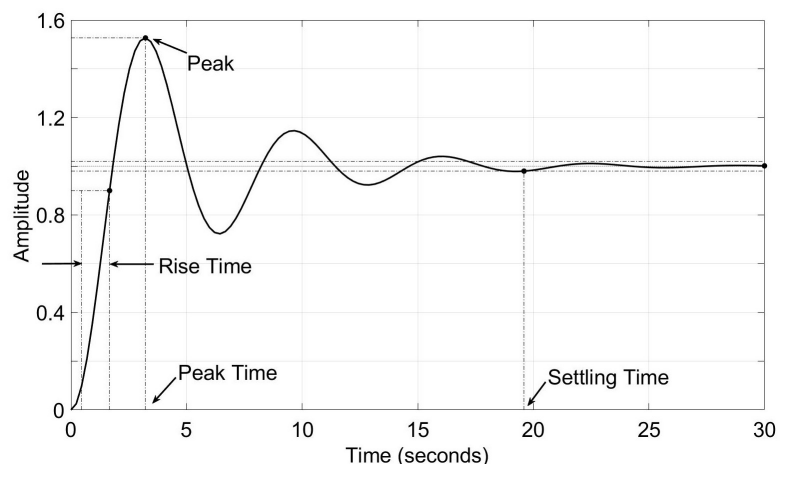

Fig. 2. Step-response characteristics of a system

The metrics for validating model-based governor response is based on the following step-response characteristics [29]:

- Peak value: Given by the peak absolute value of the governor response.

- Peak time: Given by the time-instant at which peak absolute value of the governor response occurs.

- Steady-state value: Given by the final value of the governor response.

- Rise-time: Given by the time required by the governor response to reach from $10 \%$ to $90 \%$ of it's steady-state value.

\section{ADVANCED PERFORMANCE METRICS AND THEIR APPLICATION TO THE SENSITIVITY ANALYSIS}

The methodology for model validation and calibration process has several steps:

1) Perform model validation by using play-in signal as described in [9].

2) Analyze the model validation results by comparing actual and model-based response.

3) Identify key model parameters that need to be tuned for model calibration.

4) Perform model calibration using the identified key parameters.

In this paper, the proposed methodology is to help with the second and the third step of the model validation and calibration process. The main objective of this paper is to develop metrics that can quantify mismatch between the actual and model-based response in a comprehensive and 
accurate manner, which can then facilitate the automation of the model validation process. For this, we are proposing new set of metrics in this paper that takes into consideration the mismatch corresponding to each specific aspect of generator response, such as phase and magnitude of oscillatory modes, and characteristics of the governor response. Furthermore, we are also proposing that the sensitivity analysis be performed with respect to the proposed metrics to identify key parameters for model calibration in a more systematic manner. A detailed description of the proposed methodology, shown in Fig. 3 , is discussed next.

\section{A. Step-1: Separating governor and oscillatory response}

During system faults, generator dynamic response can be broken down into two components, one is the slow governor response and the other fast oscillatory response. The generator oscillatory response is determined by system modes and therefore the frequency range of this response lies between 0.1 and $2.0 \mathrm{~Hz}$. Therefore, the slow governor response and the oscillatory response can be separated by passing the generator response through a high-pass filter having a cut-off frequency of less than $0.1 \mathrm{~Hz}$ as illustrated in the Fig. 4(a) and Fig. 4(b). The governor response is then obtained by taking the difference of the generator response and the oscillatory response, and passing the resultant signal through median filter to smooth out any oscillatory components present in the signal. This is the first and the important step in calculating proposed metrics and performing sensitivity analysis.

\section{B. Step-2: Calculation of performance metrics}

In the second step, metrics is calculated for the separated governor and oscillatory response corresponding to the active power. Metrics proposed for each of these responses is described next.

1) Active power - Oscillatory response: The metric for validating generator oscillatory response is calculated based on the properties of the oscillatory modes observed in the PMU and simulated measurements. Two metrics are proposed in this paper for validating generator oscillatory response, one quantifying magnitude similarity and the other quantifying phase similarity of oscillatory modes. The metric for magnitude incorporates any discrepancy associated with initial amplitude, damping-ratio and frequency of system modes between the model-based response and actual response. The metric for phase calculates any phase difference between the two signal. By performing sensitivity analysis with respect to these metrics can help identify model parameters that affect these parameters, and if any discrepancy is observed in either one or both of these metrics, those parameters can be tuned for model calibration.

(i) Oscillation magnitude-similarity metric $\left(O s c_{M}\right)$ : The metric for validating the magnitude component of the model-based oscillatory response is given by:

$$
O s c_{M}=1-\frac{1}{\sum_{i=1}^{p} w_{i}} \sum_{i=1}^{p} w_{i} \epsilon_{m, i}
$$

where

$$
\begin{gathered}
\epsilon_{m, i}=\left(\frac{\| \hat{\mathbf{y}}_{\mathbf{i}}^{\mathbf{a}}|-| \hat{\mathbf{y}}_{\mathbf{i}}^{\mathbf{s}}||}{\left|\hat{\mathbf{y}}_{\mathbf{i}}^{\mathbf{a}}\right|}\right) \quad \text { s.t. } 0 \leq \epsilon_{m, i} \leq 1 \\
\hat{\mathbf{y}}_{\mathbf{i}}=\left[\begin{array}{llll}
\left|\hat{y}_{i}[0]\right| & \left|\hat{y}_{i}[1]\right| & \cdots & \left.\left|\hat{y}_{i}[N-1]\right|\right]^{T} \\
\hat{y}_{i}[k]=\left|\hat{B}_{i}\right| \hat{z}_{i}^{k},
\end{array}\right.
\end{gathered}
$$

superscript ' $a$ ' corresponds to estimates using actual response and ' $s$ ' corresponds to estimates using simulated response, $\epsilon_{m, i}$ is the normalized error metric for each mode, $w_{i}$ is the weight factor for each mode given by its energy as defined in (11), $p$ is the number of dominant modes selected out of $n$ modes based on their energy, and $|$.$| denotes absolute value of the quantity. Here, the$ reconstructed signal used for calculating this metric is obtained by discarding initial phase of the modes so that the error associated with the phase does not impact the magnitude metric.

(ii) Oscillation phase-similarity metric $\left(O s c_{P}\right)$ : The metric for validating the phase component of the model-based oscillatory response is given by:

$$
O s c_{P}=1-\frac{1}{\sum_{i=1}^{p} w_{i}} \sum_{i=1}^{p} w_{i} \epsilon_{p, i}
$$

where

$$
\epsilon_{p, i}=\left(\frac{\mid\left\langle\hat{B}_{i}^{a}-\angle \hat{B}_{i}^{s}\right|}{180}\right) \quad \text { s.t. } 0 \leq \epsilon_{p, i} \leq 1,
$$

$\epsilon_{p, i}$ is the normalized phase error associated with the $i^{t h}$ mode observed in actual and simulated measurements. The metric obtained for each mode is weighted with its energy to obtain a single metric. If any mode observed in the PMU measurement is not observed in the mode estimated using the simulated data, an error of 1 is assigned to both $\epsilon_{m, i}$ and $\epsilon_{p, i}$ for that mode.

The step-wise methodology to obtain the proposed metrics for validating model-based oscillatory response is as below:

(i) Pre-process PMU and simulated measurements by using signal processing techniques, such as filtering, downsampling, etc., for modal analysis

(ii) Obtain mode estimates and mode-shapes for both preprocessed PMU and model-based measurements using (4) and (7). In this step, selection of model order is carried out for both the signals by comparing pre-processed original and reconstructed signal. Also, dominant modes are distinguished from the spurious ones by calculating energy of mode estimates using (11).

(iii) Calculate the two metrics to validate the model-based oscillatory response by comparing it with the actual oscillatory response using (12) and (16).

2) Active power - Governor response: Based on the stepresponse characteristics of a system, as shown in Fig. 2, several metrics are defined to validate the model-based governor response by comparing it with the actual governor response. Each metric looks into a specific aspect of the governor response, which are as follows: 


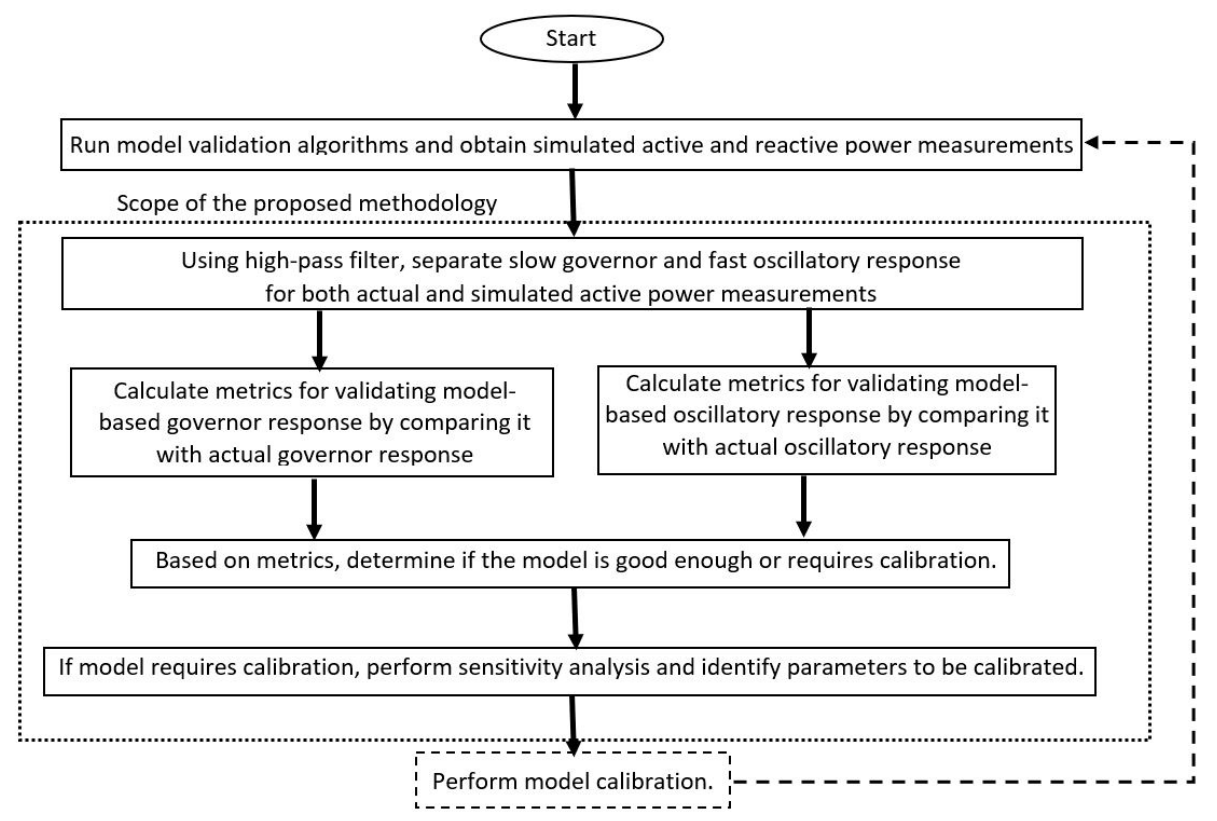

Fig. 3. FlowChart for the proposed methodology

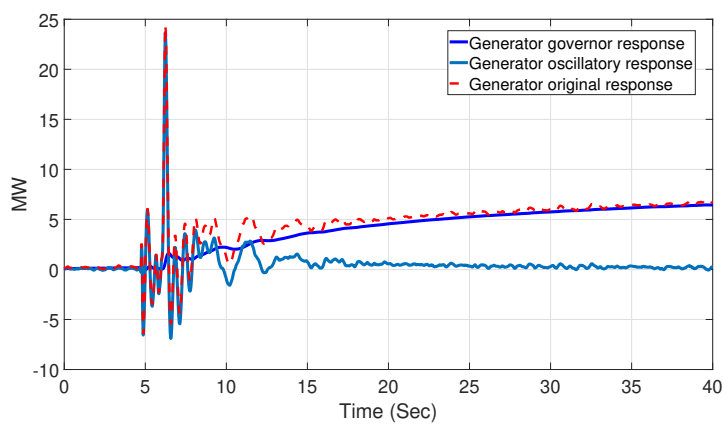

(a) Generator response and the corresponding decoupled oscillatory and governor response.

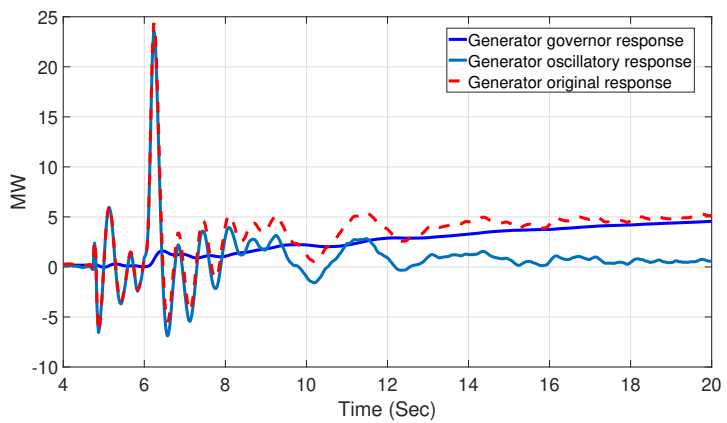

(b) Zoomed-in version of Fig. 4(a) to focus on oscillatory response.

Fig. 4. Separated governor and oscillatory response using high-pass filter.

(i) Delay $\left(G_{d}\right)$ : Obtained by taking the difference of the time taken by the model-based and actual governor response to reach $10 \%$ of their respective peak value with respect to a common time-reference.

(ii) Peak value $\left(G_{P}\right)$ : Obtained by taking the difference of the peak value of the model-based and actual governor response.

(iii) Peak time $\left(G_{P T}\right)$ : Obtained by taking the difference of the time taken by the model-based and actual governor response to reach peak-value

(iv) Steady-state error $\left(G_{S S}\right)$ : Obtained by taking the difference of the final value of the model-based and actual governor response

(v) Rise time $\left(G_{R T}\right)$ : Obtained by taking the difference of the time taken by the model-based and actual governor response to change from $20 \%$ to $90 \%$ of their respective peak-value.

Ideally, the mismatch observed in the actual and modelbased generator response should be equal to zero. However, that is generally not the case. Therefore, certain thresholds need to be determined for each metric, summarized in Table I, to validate the generator model. These thresholds should be determined based on the industry practices and is beyond the scope of the paper.

TABLE I

PROPOSED METRICS FOR QUANTIFYING MISMATCH BETWEEN ACTUAL AND MODEL-BASED RESPONSE

\begin{tabular}{|l|c|c|c|c|c|}
\hline Oscillatory response & $O s c_{M}$ & $O s c_{P}$ & \multicolumn{3}{|c|}{} \\
\hline Governor response & $G_{d}$ & $G_{P}$ & $G_{P T}$ & $G_{R T}$ & $G_{S S}$ \\
\hline
\end{tabular}

C. Step-3: Identifying key parameters by performing sensitivity analysis with respect to the proposed metrics

Based on the calculated metrics, if it is determined that the model needs calibration then the next step will be to perform sensitivity analysis to identify key parameters that need to be tuned for model calibration. In this paper, we are proposing that the sensitivity analysis be carried out with respect to the proposed metrics that quantifies the impact of each model parameter on specific error metrics that have mismatch between actual and model-based response. For example, if it is identified that the generator model response has mismatch with the actual response for governor response time, given by delay metric, then the sensitivity analysis needs to be performed with 
respect to the delay metric to identify parameters that have significant impact on delay metric as given by:

$$
S_{G_{d}, H}=\frac{\Delta G_{d}}{\Delta H} .
$$

Depending on how the selected model parameter affects other metrics, other parameters may also have to be selected.

\section{RESUlts AND Discussions}

Results were obtained using both simulated and real-world PMU measurements based case-studies to illustrate the effectiveness of the proposed metrics and their application to the sensitivity analysis.

\section{A. Simulated data based case-study}

The simulated-data based example used in this paper is taken from the 12 disturbances set prepared by NASPI Engineering Analysis Task Team and NERC synchrophasor measurement subcommittee team for NASPI Technical Workshop on Model Verification Tools in 2016 [30]. Fig. 5 shows the active power measured at the POI of the generator, and the model-based response of the generator obtained using PPMV tool developed by BPA and PNNL. Fig. 6(a) and Fig. 6(b) show governor and oscillatory response obtained from actual and model-based active power response. The results obtained for oscillatory and governor response is presented next. For comparison purpose, a normalized root mean square error metric was also calculated using

$$
N . R M S E=\sqrt{\frac{1}{N} \sum_{k=1}^{N} \epsilon_{k}^{2},}
$$

where

$$
\epsilon_{k}=\left|\frac{y^{a}[k]-y^{s}[k]}{y^{a}[k]}\right| \quad \text { s.t. } 0 \leq \epsilon_{k} \leq 1
$$

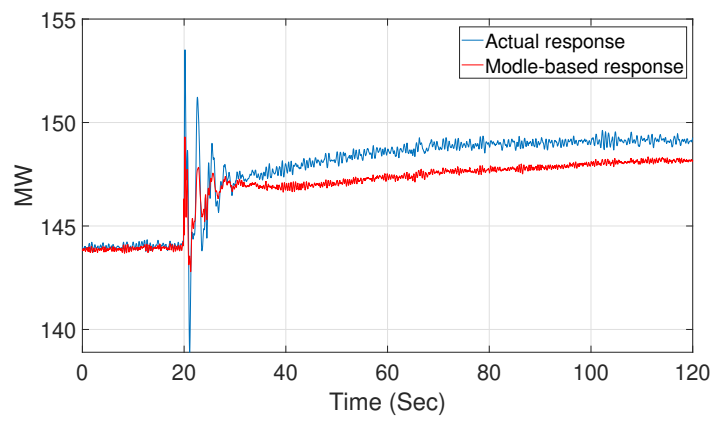

Fig. 5. PMU measurements recorded at the Point of Interconnection, and model-based response of the generator obtained using PPMV tool

1) Metrics for oscillatory response: Using the methodology described in the earlier section, metrics were calculated for validating the model-based oscillatory response of the generator. Before performing modal analysis, the signals were downsampled to $5 \mathrm{samples} / \mathrm{sec}$ and also frequency components lower than $0.1 \mathrm{~Hz}$ were removed. Using this pre-processed

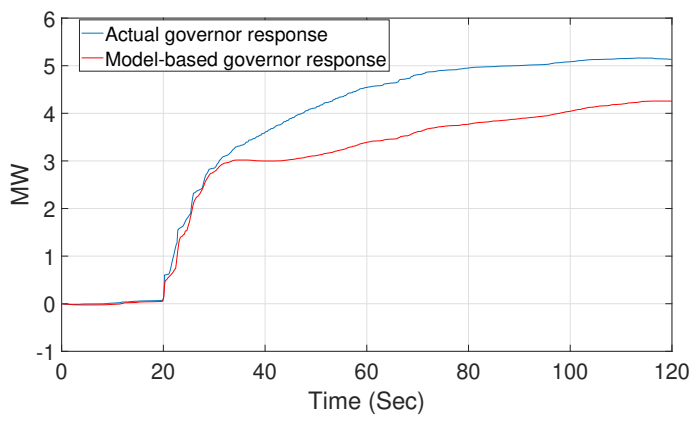

(a) Generator governor response

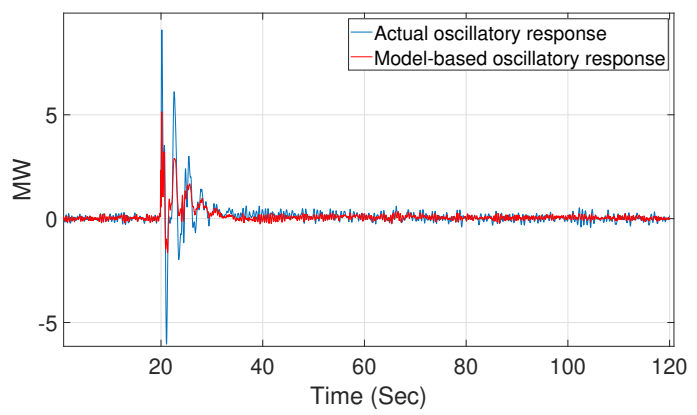

(b) Generator oscillatory response

Fig. 6. Generator governor and oscillatory response calculated using actual and model-based active power response.

measurements, system modes and mode shapes were estimated for both actual measurements and model-based response. The model order selection is very critical to the proposed method as it can significantly affect the metrics for quantifying the validation results. For both actual measurements and modelbased simulated data, model order of $n=22$ was chosen that gave the best fit between the original and reconstructed signal as shown in Fig. 7(a) and Fig 7(b).

Table II and III give the mode estimates for the PMU and model-based simulated measurements. For metric calculations, mode estimates having energy less than $5 \%$ of the highest energy were not considered.

TABLE II

Mode estimates For PMU MEASUREMENTS

\begin{tabular}{|c|c|c|c|c|}
\hline $\begin{array}{l}\text { Frequency } \\
(\mathrm{Hz})\end{array}$ & $\begin{array}{l}\text { Damping } \\
\text { ratio (\%) }\end{array}$ & $\begin{array}{l}\text { Initial } \\
\text { Amplitude }\end{array}$ & $\begin{array}{l}\text { Initial } \\
\text { Phase (Deg) }\end{array}$ & $\begin{array}{l}\text { Normalized } \\
\text { Energy }\end{array}$ \\
\hline 0.362 & 11.999 & 3.028 & 48.384 & 1.000 \\
\hline 0.799 & 14.678 & 2.990 & -65.998 & 0.426 \\
\hline 0.634 & 8.873 & 1.599 & 161.012 & 0.272 \\
\hline 1.248 & 2.276 & 0.503 & 64.129 & 0.045 \\
\hline 1.737 & 1.177 & 0.412 & -33.676 & 0.040 \\
\hline
\end{tabular}

TABLE III

MOdE ESTIMATES FOR MODEL-BASED SIMULATED DATA

\begin{tabular}{|c|c|c|c|c|}
\hline $\begin{array}{l}\text { Frequency } \\
(\mathrm{Hz})\end{array}$ & $\begin{array}{l}\text { Damping } \\
\text { ratio (\%) }\end{array}$ & $\begin{array}{l}\text { Initial } \\
\text { Amplitude }\end{array}$ & $\begin{array}{l}\text { Initial } \\
\text { Phase (Deg) }\end{array}$ & $\begin{array}{l}\text { Normalized } \\
\text { Energy }\end{array}$ \\
\hline 0.361 & 11.759 & 1.236 & 41.543 & 1.000 \\
\hline 0.814 & 12.912 & 1.177 & -76.053 & 0.399 \\
\hline 1.935 & 4.673 & 0.545 & 30.661 & 0.126 \\
\hline 0.634 & 8.592 & 0.419 & 166.861 & 0.111 \\
\hline 2.038 & 10.432 & 0.758 & 151.799 & 0.101 \\
\hline 1.749 & 4.239 & 0.351 & -1.507 & 0.059 \\
\hline 1.261 & 1.953 & 0.112 & 13.848 & 0.015 \\
\hline
\end{tabular}

Using (12) and (16), metrics for validating model-based oscillatory response was calculated and is given in Table IV. 


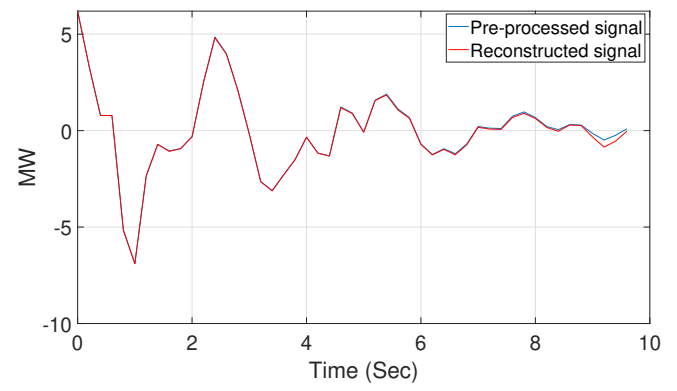

(a) For PMU measurements: $n=22$ and Goodness of fit $=0.96$

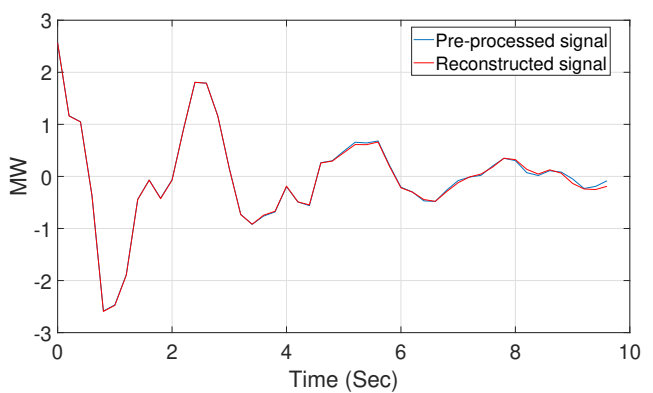

(b) For Model-based response: $n=22$ and Goodness of fit $=0.97$

Fig. 7. Illustration of the model order selection by comparing pre-processed signal with the reconstructed signal.

Based on the oscillation magnitude-similarity metric, it can be said that the dynamic model does not accurately represent the model that generated the PMU measurements and requires calibration. This is also illustrated in Fig. 8(a) and 8(b) that compare the contribution of two dominant mode estimates to the PMU measurements and simulated generator response. As seen in these figures, the contribution of the two modes to the PMU measurements and generator response do not have a good match. However, the phase component of the oscillatory response matched well based on the calculated oscillation phase-similarity metric. By performing sensitivity analysis, model parameters that affected the magnitude of the oscillatory response were identified as discussed in later section.

TABLE IV

METRICS CALCULATED FOR OSCILLATORY RESPONSE.

\begin{tabular}{|l|c|c|c|c|c|}
\hline & Mode-1 & Mode-2 & Mode-3 & Mode-4 & \multirow{2}{*}{ Osc. Metric } \\
\hline$w_{i}$ & 1 & 0.426 & 0.272 & 0.045 & \\
\hline$\epsilon_{m, i}$ & 0.588 & 0.593 & 0.734 & 0.772 & 0.3759 \\
\hline$\epsilon_{p, i}$ & 0.038 & 0.056 & -0.032 & 0.279 & 0.9342 \\
\hline
\end{tabular}

2) Metrics for governor response: Using governor response extracted from actual and model-based response measurements, metrics were calculated comparing the actual and model-based governor response and are given in Table V. Based on these metrics, it can be said that model parameters that can increase the peak-value of the governor response needs to be calibrated. These parameters were identified using sensitivity analysis as discussed in the later section.

TABLE V

METRICS CALCULATED FOR GOVERNOR RESPONSE

\begin{tabular}{|c|c|c|c|c|}
\hline$G_{d}(\mathrm{sec})$ & $G_{P}(\mathrm{MW})$ & $G_{P T}(\mathrm{sec})$ & $G_{R T}(\mathrm{sec})$ & $G_{S S}(\mathrm{MW})$ \\
\hline 0.025 & 0.9 & -4 & -18.32 & 0.88 \\
\hline
\end{tabular}

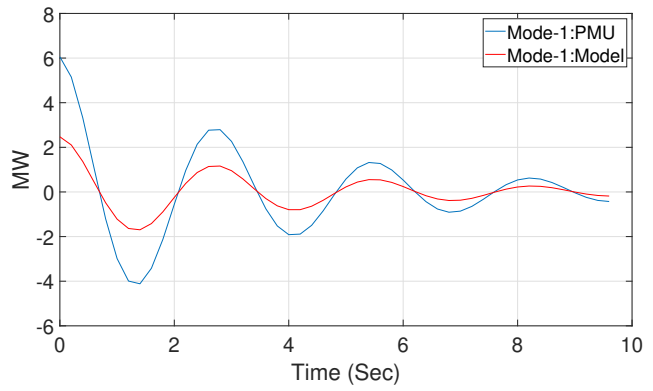

(a) Mode-1

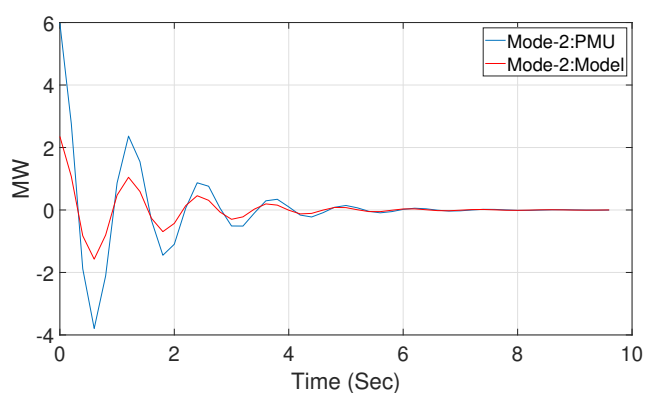

(b) Mode-2

Fig. 8. Comparison of contribution of selected modes to the magnitude component of oscillatory response of actual and model-based response

3) Comparison with the RMSE metric: For comparison purpose, a normalized RMSE metric was calculated using (19), and is equal to $0.39642>>0$ indicating poor model quality. However, as previously mentioned, the information provided by this metric is limited as compared to that by the proposed set of metrics which can help with the automation of model validation process without losing any information and also with the identification of key model parameters to be tuned.

\section{B. Real-world data based case-study}

The proposed methodology was also implemented using real PMU measurements recorded in the Western Interconnection. The allowable error limits for synchrophasor measurements and minimum sampling frequency, as defined in the IEEE/IEC Standard 60255-118-1-2018 [31], meets the criteria for using synchrophasor measurements for model validation and calibration.

Fig. 9 shows the comparison of the actual and model-based active-power response. The methodology described earlier were used to obtain the metrics, which are shown in Fig. 10 (a) and 10(b), along with the respective responses. For modal analysis, model order of $n=18$ was chosen for both actual and model-based response with $G o F$ metric equal to 0.99 and 1.00 respectively. Table VI provides the error associated with each mode of the oscillatory response. Based on the response obtained for the governor response, it can be seen that the mismatch between the actual and modelbased response comes from delay and peak value, which could not have been known by using a simple RMSE metric. Based on the oscillation magnitude-similarity metric, it can be said that the model parameters need to be calibrated to improve oscillatory magnitude response of the model. Using (19), the normalized RMSE for this example was calculated to 


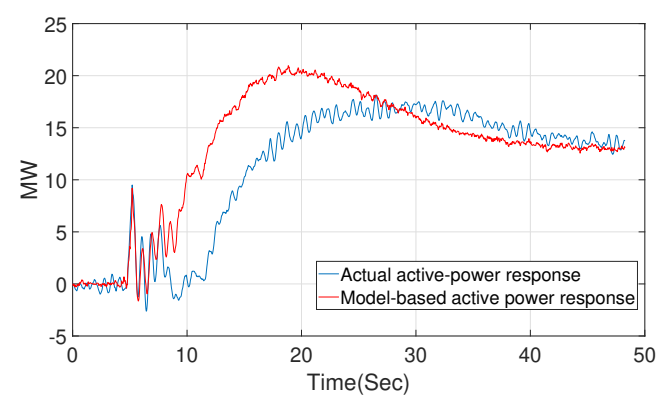

Fig. 9. Real-world PMU measurements and model-based response

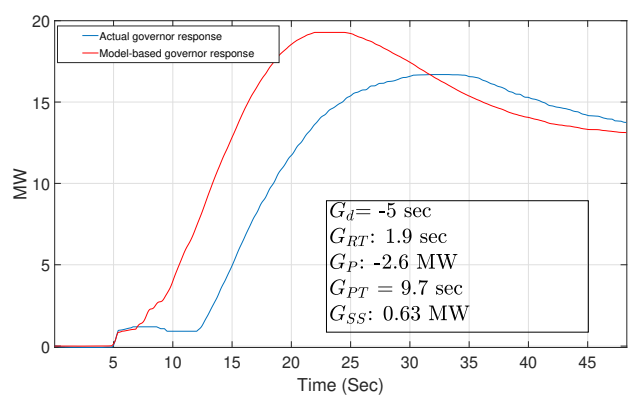

(a) Actual and model-based governor response

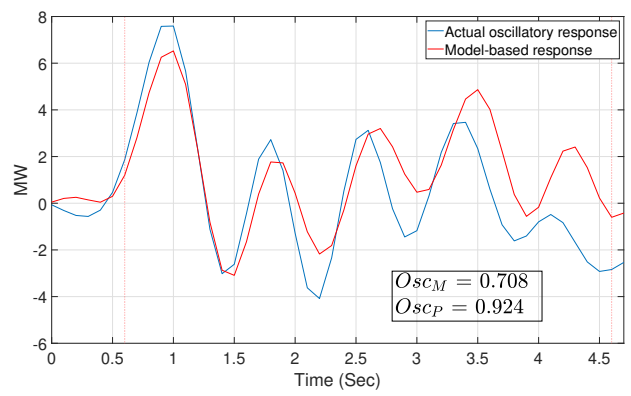

(b) Actual and model-based oscillatory response

Fig. 10. Metrics calculated for the real-world data example.

be $0.5382>>0$ indicating poor model quality. Again, if the model validation process is to be automated using this simple RMSE metric, some critical information would be lost which can be helpful for identifying key model parameters. Based on the calculated metrics, model parameters to be tuned can be identified by performing sensitivity analysis with respect to the governor peak-value metric, governor delay metric and oscillatory magnitude-similarity metric. However, for this example, sensitivity analysis was not performed as the point of interconnection at which the measurements were recorded consists of multiple units.

TABLE VI

METRICS FOR OSCILLATORY RESPONSE REAL-WORLD DATA EXAMPLE.

\begin{tabular}{|c|c|c|c|}
\hline & Mode-1 & Mode-2 & \multirow{2}{*}{ Osc. Metric } \\
\hline$w_{i}$ & 1.000 & 0.457 & \\
\hline$\epsilon_{m, i}$ & 0.341 & 0.105 & 0.708 \\
\hline$\epsilon_{p, i}$ & 0.093 & 0.01 & 0.924 \\
\hline
\end{tabular}

TABLE VII

MODEL PARAMETERS FOR SIMULATED-DATA BASED CASE STUDY EXAMPLE

\begin{tabular}{|l|r|l|r|l|r|l|r|}
\hline \multicolumn{2}{|c|}{ GENROU } & \multicolumn{2}{|c|}{ REXS } & \multicolumn{2}{c|}{ GGOV1 } & \multicolumn{2}{c|}{ PSS2A } \\
\hline Param & Value & Param & Value & Param & Value & Param & Value \\
\hline Xd & 1.85 & Tr & 0.02 & R & 0.05 & J1 & 1 \\
\hline X'd & 0.21 & Kvp & 600 & Rselect & 1 & K1 & 0 \\
\hline X'd & 0.15 & Kvi & 0 & Tpelec & 0.6 & J2 & 3 \\
\hline Xq & 1.3 & Vimax & 0.2 & Maxerr & 0.025 & K2 & 0 \\
\hline X'q & 0.7 & Ta & 0.02 & Minerr & -0.025 & Tw1 & 1 \\
\hline X'q & 0.15 & Tb1 & 1 & Kpgov & 6 & Tw2 & 1 \\
\hline Xl & 0.15 & Tc1 & 10 & Kigov & 0.22 & T3 & 5 \\
\hline Ra & 0.003 & Tb2 & 1 & Kdgov & 0 & Tw4 & 0 \\
\hline T'do & 5 & Tc2 & 1 & Tdgov & 1 & T6 & 0 \\
\hline T'do & 0.25 & Vrmax & 10 & Vmax & 1 & T7 & 5 \\
\hline T'qo & 1 & Vrmin & -10 & Vmin & 0.24 & Ks2 & 0.5 \\
\hline T'qo & 0.05 & Kf & 0.045 & Tact & 0.6 & Ks3 & 1 \\
\hline S(1.0) & 0.12 & Tf & 5 & Kturb & 1.5 & Ks4 & 1 \\
\hline S(1.2) & 0.48 & Tf1 & 1 & Wfnl & 0.25 & T8 & 0.5 \\
\hline H & 3.1 & Tf2 & 1 & Tb & 1 & T9 & 0.1 \\
\hline D & 0 & Fbf & 1 & Tc & 1 & N & 1 \\
\hline Rcomp & 0 & Kip & 5 & Flag & 1 & M & 5 \\
\hline Xcomp & 0 & Kii & 0 & Teng & 0 & Ks1 & 15 \\
\hline Accel & 0.5 & Tp & 0 & Tfload & 0.3 & T1 & 0.28 \\
\hline Kis & 0 & Vfmax & 99 & Kpload & 1 & T2 & 0.043 \\
\hline Pfd & 0 & Vfmin & -99 & Kiload & 3.3 & T3 & 0.281 \\
\hline Pkd & 0 & Kh & 0 & Ldref & 1 & T4 & 1.16 \\
\hline Pfq & 0 & Ke & 0.4 & Dm & 0 & Vstmax & 0.1 \\
\hline Pkq & 0 & Te & 1.2 & Ropen & 99 & Vstmin & -0.1 \\
\hline Speed & 0 & Kc & 0 & Rclose & -99 & A & 1 \\
\hline Angle & 0 & Kd & 0.7 & Kimw & 0 & Ta & 0 \\
\hline & & E1 & 2.4 & Pmwest & 0 & Tb & 0.043 \\
\hline & & Se1 & 0.05 & Asest & 99 & & \\
\hline & & E2 & 3.2 & Ka & 10 & & \\
\hline & & Se2 & 0.3 & Tact & 1 & & \\
\hline & & Rcomp & 0 & Db & 0 & & \\
\hline & & Xcomp & 0 & Tsa & 1 & & \\
\hline & & 0 & Tsb & & & & \\
\hline & & & & & & & \\
\hline & & & & & & & \\
\hline & & & & & & \\
\hline & & & & & & \\
\hline & & & & & & \\
\hline & & & & & & \\
\hline & & & & & & \\
\hline & & & & & & \\
\hline & & & & & & \\
\hline
\end{tabular}

C. An example illustrating the application of proposed metrics to the sensitivity analysis and model calibration

In this paper, simulated-data based example is further used to illustrate the application of proposed metrics for sensitivity analysis. The model parameters of the generator machine models and governor models in this case-study example is shown in Table VII. In this example, all model parameters were changed by certain $\%$ and the corresponding change in the error metrics were calculated with respect to the original error metric using

$$
\Delta E=E_{\text {new }}-E_{\text {old }},
$$

where $E$ is the error metric for which sensitivity analysis was carried out. The sensitivity analysis results for the generator machine model (GENROU) parameters are summarized in Table VIII. Table IX provides sensitivity analysis results for the selected parameters of the governor model (GGOV1) that were significant. The change in the exciter and stabilizer model parameters did not result in any significant changes 
in the active power response and therefore the results are not included. For comparison purpose, the sensitivity analysis results are also included with respect to the RMSE metric in Table VIII and Table IX.

In this example, based on the proposed metrics, calibration of model is required to improve oscillatory similarity metric and governor peak-value metric. Based on the sensitivity analysis with respect to the proposed metrics, we were able to identify parameters that can improve specific aspect of generator response that has mismatch with the actual response, for example inertia constant $\mathrm{H}$ to improve oscillatory magnitude similarity metric and integral gain (kigov) and proportional gain (kpgov) for improving governor response. If we perform sensitivity analysis with respect to the RMSE metric, then we will end up selecting several parameters that will affect several aspects of dynamic response, for example parameters such as $\mathrm{H}, \mathrm{Xd}, \mathrm{X}$ 'q, and T'do of machine model which affect both oscillatory magnitude and phase similarity metric. Therefore, performing sensitivity analysis with respect to the proposed metrics can help narrow down parameters to be calibrated in a more systematic and scientific manner.

TABLE VIII

SENSITIVITY ANALYSIS RESULT FOR MACHINE MODEL PARAMETERS

\begin{tabular}{|l|r|r|r|r|r|r|r|r|r|}
\hline Param. & $\%$ change & $\Delta O s c_{M}$ & $\Delta O s c_{P}$ & $\Delta G_{d}$ & $\Delta G_{P}$ & $\Delta G_{P T}$ & $\Delta G_{R T}$ & $\Delta G_{S S}$ & $N . R M S E$ \\
\hline Metric & & 0.37 & 0.93 & 0 & 0.9 & -4 & -18.32 & 0.88 & 0.396 \\
\hline $\mathrm{H}$ & $50 \%$ & 0.35 & 0.02 & 0.0 & 0.0 & 0 & 0.6 & 0.0 & -0.0082 \\
\hline $\mathrm{H}$ & $-50 \%$ & -0.17 & 0.02 & 0.0 & 0.0 & 0 & -0.4 & 0.0 & 0.0161 \\
\hline $\mathrm{Xd}$ & $50 \%$ & 0.00 & 0.01 & 0.0 & 0.0 & 0 & 0.2 & 0.0 & -0.0078 \\
\hline $\mathrm{Xd}$ & $-50 \%$ & -0.04 & -0.06 & 0.0 & 0.0 & 0 & 0.1 & 0.0 & 0.0160 \\
\hline X'd & $50 \%$ & -0.04 & -0.06 & 0.0 & 0.0 & 0 & -0.1 & 0.0 & 0.0166 \\
\hline X'd & $-50 \%$ & -0.01 & -0.04 & 0.0 & 0.0 & 0 & 0.0 & 0.0 & 0.0042 \\
\hline X'q & $50 \%$ & -0.05 & -0.04 & 0.0 & 0.0 & 0 & -0.2 & 0.0 & 0.0006 \\
\hline X'q & $-50 \%$ & 0.06 & 0.18 & 0.0 & 0.0 & 0 & 0.0 & 0.0 & 0.0131 \\
\hline Xq & $50 \%$ & 0.05 & 0.18 & 0.0 & 0.0 & 0 & 0.0 & 0.0 & 0.0128 \\
\hline Xq & $-50 \%$ & -0.04 & -0.03 & 0.0 & 0.0 & 0 & 0.2 & 0.0 & -0.0034 \\
\hline T'do & $50 \%$ & -0.05 & -0.05 & 0.0 & 0.0 & 0 & 0.1 & 0.0 & -0.0028 \\
\hline T'do & $-50 \%$ & 0.00 & 0.00 & 0.0 & 0.0 & 0 & 0.1 & 0.0 & 0.0000 \\
\hline T'do & $50 \%$ & -0.05 & -0.05 & 0.0 & 0.0 & 0 & 0.2 & 0.0 & -0.0098 \\
\hline T'do & $-50 \%$ & -0.03 & 0.00 & 0.0 & 0.0 & 0 & 0.1 & 0.0 & 0.0113 \\
\hline T"qo & $50 \%$ & -0.01 & 0.00 & 0.0 & 0.0 & 0 & 0.2 & 0.0 & -0.0043 \\
\hline T'qo & $-50 \%$ & -0.05 & -0.03 & 0.0 & 0.0 & 0 & 0.0 & 0.0 & 0.0036 \\
\hline T'qo & $50 \%$ & -0.02 & -0.01 & 0.0 & 0.0 & 0 & 0.1 & 0.0 & 0.0011 \\
\hline T'qo & $-50 \%$ & -0.05 & -0.03 & 0.0 & 0.0 & 0 & 0.2 & 0.0 & -0.0001 \\
\hline
\end{tabular}

TABLE IX

SENSITIVITY ANALYSIS RESULT FOR GOVERNOR MODEL PARAMETERS

\begin{tabular}{|l|r|r|r|r|r|r|r|r|r|}
\hline Param. & \%change & $\Delta O s c_{M}$ & $\Delta O s c_{P}$ & $\Delta G_{d}$ & $\Delta G_{P}$ & $\Delta G_{P T}$ & $\Delta G_{R T}$ & $\Delta G_{S S}$ & $N . R M S E$ \\
\hline Metric & & 0.37 & 0.93 & 0 & 0.9 & -4 & -18.32 & 0.88 & 0.396 \\
\hline kigov & $50 \%$ & 0.0 & 0.0 & 0.0 & 0.56 & -2.7 & -13.93 & 0.54 & -0.036 \\
\hline kigov & $-50 \%$ & 0.0 & 0.1 & 0.0 & -1.03 & 0.5 & 8.48 & -1.04 & 0.095 \\
\hline kpgov & $50 \%$ & 0.0 & 0.0 & 0.0 & -0.05 & -2.2 & -55.21 & -0.08 & 0.005 \\
\hline kpgov & $-50 \%$ & 0.0 & 0.0 & 0.7 & 0.13 & 2.0 & 4.59 & 0.13 & 0.019 \\
\hline kturb & $25 \%$ & 0.0 & 0.0 & 0.0 & 0.28 & -0.4 & -11.49 & 0.26 & -0.021 \\
\hline kturb & $-25 \%$ & 0.1 & 0.0 & 0.7 & -3.57 & -84.7 & -61.63 & -4.57 & 0.569 \\
\hline R & $50 \%$ & 0.0 & 0.1 & 0.0 & -1.12 & -2.7 & -14.20 & -1.15 & 0.090 \\
\hline $\mathrm{R}$ & $-50 \%$ & 0.0 & 0.0 & 0.0 & 2.19 & 2.0 & 12.50 & 2.19 & -0.024 \\
\hline
\end{tabular}

Based on this sensitivity analysis results, model calibration was carried out by manually tuning key model parameters identified using sensitivity analysis with respect to the proposed metrics. By increasing the value of inertia-constant $\mathrm{H}$, the magnitude component of the oscillatory response showed a better match with the actual oscillatory response. For improving governor response, first the integral gain (kigov) was increased and then the proportional gain (kpgov) was adjusted to minimize error associated with the peak-time. The modelbased active power response obtained using this calibrated model is shown in Fig. 11 along with the error metrics. As can be seen in this figure, the error metrics is much improved as compared to the original model. One thing must be noted here that, the objective of this example is only to illustrate how these proposed metrics can be helpful for model validation and calibration, and not model calibration itself.

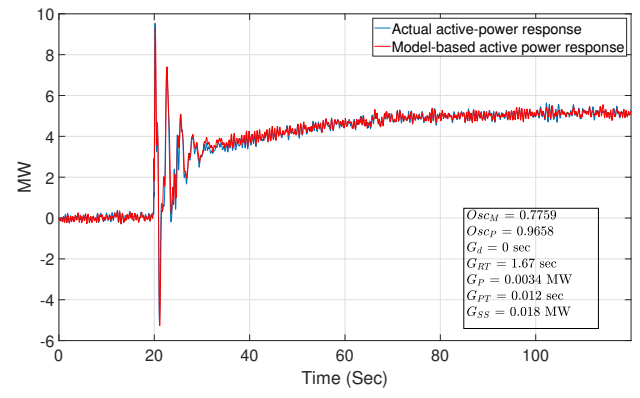

Fig. 11. Model calibration using proposed metrics and sensitivity analysis

\section{CONClusion}

This paper proposes advanced performance metrics for quantifying model validation results in a rigorous manner by breaking down generator active power response into slow governor response and fast oscillatory response. These proposed metrics analyze several aspects of generator dynamic response as compared to other existing metrics, and therefore provides more accurate and comprehensive assessment of model validation results. The results obtained using both simulated and real-world measurements based case-studies validate the effectiveness of the proposed metrics to help with the automation of the model validation process without having to lose any information captured by visual inspection. Furthermore, performing sensitivity analysis with respect to the proposed metrics can identify key parameters in a more systematic and scientific manner that are sensitive to specific error metrics having significant mismatch between actual and model-based response. This has been illustrated in the paper using simulated test-case based example. To conclude, proposed metrics and sensitivity analysis can be very useful for model validation and calibration. Future work will focus on advancing the use of proposed metrics for model calibration and also combining metrics for several events into a single metric that can help with the model calibration based on multiple events.

\section{REFERENCES}

[1] D. N. Kosterev, C. W. Taylor, and W. A. Mittelstadt, "Model validation for the august 10, 1996 WSCC system outage," IEEE transactions on power systems, vol. 14, no. 3, pp. 967-979, 1999.

[2] Standard MOD-026-1 - Verification of Models and Data for Generator Excitation Control System or Plant Volt/Var Control Functions., NERC Std.

[3] Standard MOD-027-1 - Verification of Models and Data for Turbine/Governor and Load Control or Active Power/Frequency., NERC Std.

[4] J. Feltes and L. Lima, "Validation of dynamic model parameters for stability analysis; industry need, current practices and future trends," in 2003 IEEE Power Engineering Society General Meeting (IEEE Cat. No. 03CH37491), vol. 3. IEEE, 2003, pp. 1295-1301. 
[5] J. Undrill, L. Pereira, D. Kosterev, S. Patterson, D. Davies, S. Yang, and B. Agrawal, "Generating unit model validation: WECC lessons and moving forward," in 2009 IEEE Power \& Energy Society General Meeting. IEEE, 2009, pp. 1-5.

[6] P. Overholt, D. Kosterev, J. Eto, S. Yang, and B. Lesieutre, "Improving reliability through better models: Using synchrophasor data to validate power plant models," IEEE Power and Energy Magazine, vol. 12, no. 3, pp. 44-51, May 2014.

[7] M. Wu, W. Huang, F. Q. Zhang, X. Luo, S. Maslennikov, and E. Litvinov, "Power plant model verification at ISO new england," in 2017 IEEE Power \& Energy Society General Meeting. IEEE, 2017, pp. 1-5.

[8] M. Wu and L. Xie, "A feature-based diagnosis framework for power plant model validation," in 2018 Power Systems Computation Conference (PSCC). IEEE, 2018, pp. 1-7.

[9] Z. Huang, M. Kosterev, R. Guttromson, and T. Nguyen, "Model validation with hybrid dynamic simulation," in 2006 IEEE Power Engineering Society General Meeting. IEEE, 2006, pp. 9-pp.

[10] P. Pourbeik, "Automated parameter derivation for power plant models from system disturbance data," in 2009 IEEE Power \& Energy Society General Meeting. IEEE, 2009, pp. 1-10.

[11] J. F. Hauer, M. J. Beshir, and W. A. Mittelstadt, "Dynamic performance validation in the western power system," Association of Power Exchanges, 2000.

[12] D. Kosterev, "Hydro turbine-governor model validation in pacific northwest," IEEE Transactions on Power Systems, vol. 19, no. 2, pp. 1144 1149, 2004.

[13] Y. Li, R. Diao, R. Huang, P. Etingov, X. Li, Z. Huang, S. Wang, J. Sanchez-Gasca, B. Thomas, M. Parashar, G. Pai, S. Kincic, and A. Ning, "An innovative software tool suite for power plant model validation and parameter calibration using PMU measurements," in 2017 IEEE Power Energy Society General Meeting, July 2017, pp. 1-5.

[14] N. Nayak, H. Chen, W. Schmus, and R. Quint, "Generator parameter validation and calibration process based on PMU data," in 2016 IEEE/PES Transmission and Distribution Conference and Exposition $(T \& D)$. IEEE, 2016, pp. 1-5.

[15] W. Ju, N. Nayak, C. Vikram, H. Silva-Saravia, K. Sun, and G. Zu, "Indices for automated identification of questionable generator models using synchrophasors," in 2020 IEEE Power \& Energy Society General Meeting (PESGM). IEEE, 2020, pp. 1-5.

[16] N. Nayak. (2019, Sep.) Generator parameter validation (GPV). Electric Power Group (EPG). [Online]. Available: https://electricpowergroup. app.box.com/v/EPGWebinar4Presentation

[17] H. Wang, P. Wang, A. Menon, M. Parashar, K. Srinivasan, S. Chen, and R. Markham, "Feature adaptive generator model calibration," in 2020 IEEE Power \& Energy Society General Meeting (PESGM). IEEE, 2020, pp. $1-5$.

[18] C.-C. Tsai, L.-R. Chang-Chien, I.-J. Chen, C.-J. Lin, W.-J. Lee, C.C. Wu, and H.-W. Lan, "Practical considerations to calibrate generator model parameters using phasor measurements," IEEE Transactions on Smart Grid, vol. 8, no. 5, pp. 2228-2238, 2016.

[19] K. Shin, "An alternative approach to measure similarity between two deterministic transient signals," Journal of Sound and Vibration, vol. 371, pp. 434-445, 2016.

[20] E. Rezaei and M. V. Venkatasubramanian, "How well does a model simulation match with system response?" North American SynchroPhasor Initiative, 2019.

[21] E. Rezaei and V. Venkatasubramanian, "Quantitative indicators for quality of fit assessment in power system model validation problems," in 2018 IEEE International Symposium on Circuits and Systems (ISCAS). IEEE, 2018, pp. 1-5.

[22] U. Agrawal, P. Etingov, and R. Huang, "Initial results of quantification of model validation results using modal analysis," in 2020 IEEE Power \& Energy Society General Meeting. IEEE, 2020, pp. 1-5.

[23] X. Cheng, W.-J. Lee, M. Sahni, Y. Cheng, and L. K. Lee, "Dynamic equivalent model development to improve the operation efficiency of wind farm," in 2015 IEEE/IAS 51st Industrial \& Commercial Power Systems Technical Conference (I\&CPS). IEEE, 2015, pp. 1-9.

[24] Y. Wang, C. Lu, L. Zhu, G. Zhang, X. Li, and Y. Chen, "Comprehensive modeling and parameter identification of wind farms based on wide-area measurement systems," Journal of Modern Power Systems and Clean Energy, vol. 4, no. 3, pp. 383-393, 2016.

[25] R. Huang, R. Diao, Y. Li, J. Sanchez-Gasca, Z. Huang, B. Thomas, P. Etingov, S. Kincic, S. Wang, R. Fan et al., "Calibrating parameters of power system stability models using advanced ensemble kalman filter," IEEE Transactions on Power Systems, vol. 33, no. 3, pp. 2895-2905, 2017.
[26] J. F. Hauer, C. J. Demeure, and L. L. Scharf, "Initial results in prony analysis of power system response signals," IEEE Transactions on Power Systems, vol. 5, no. 1, pp. 80-89, Feb 1990.

[27] T. K. Sarkar and O. Pereira, "Using the matrix pencil method to estimate the parameters of a sum of complex exponentials," IEEE Antennas and Propagation Magazine, vol. 37, no. 1, pp. 48-55, 1995.

[28] U. Agrawal and J. W. Pierre, "Visual validation of estimated parametric models of power systems under ambient conditions," in Proceedings of the 52nd Hawaii International Conference on System Sciences, 2019.

[29] The MathWorks, Inc., Control System Toolbox Reference ${ }^{T M}$ (R2020a), Natick, Massachusetts, United State, 2020. [Online]. Available: https://www.mathworks.com/help/pdf_doc/control/control_ref.pdf

[30] R. Quint and D. Ramasubramanian. (2016, Oct.) PPMV Tools Calibration Session Simulations. NASPI-NERC Workshop. [Online]. Available: https://www.naspi.org/sites/default/files/2017-03/ 201610 NASPI 03 Calibration Session.pdf

[31] IEEE/IEC 60255-118-1-2018 - Synchrophasor for power systems Measurements, Std., 2018.

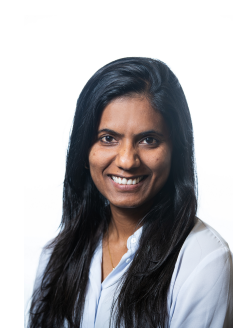

Urmila Agrawal (S'18-M'19) received the B.E. degree in electrical engineering from Pulchowk Campus, Kathmandu, Nepal, in 2011, the M.S. degree in electrical engineering and the Ph.D. degree in electrical engineering with a minor in statistics from the University of Wyoming, Laramie, WY, USA, in 2014 and 2018, respectively. She worked as an Electrical Design Engineer from 2011 to 2012 with Hulas Engineering \& Construction Pvt. Ltd., Kathmandu. She joined Pacific Northwest National Laboratory (PNNL) in 2018 as a Power Systems Research Engineer. Her research interests include application of statistical signal processing to power systems, power systems resiliency and reliability studies, power system modeling and validation, wide area monitoring and situational awareness, renewable integration studies, etc.

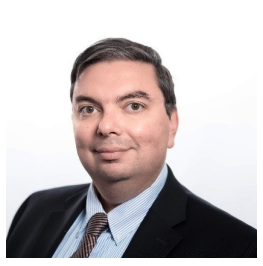

Pavel Etingov (M'05) is a staff research engineer at the US Department of Energy's Pacific Northwest National Laboratory (PNNL), Richland, WA. He graduated with honors from Irkutsk State Technical University specializing in electrical engineering in 1997. He was a fellow at the Swiss Federal Institute of Technology in 2000-2001. P.V. Etingov received his Ph.D. degree in 2003 from the Energy Systems Institute of the Russian Academy of Sciences, Irkutsk, Russia. He joined PNNL in 2008 where he serves as a Project Manager, Principal Investigator (PI)/co-PI and key technical contributor in multiple projects. He is a member of the IEEE Power \& Energy Society (PES), CIGRE, WECC Joint Synchronized Information Subcommittee (JSIS), WECC Modeling and Validation Work Group (MVWG), and North American SynchroPhasor Initiative (NASPI) research analysis task team. His research interests include stability analysis of electric power systems, power system operation, modeling and control, phasor measurement units (PMUs) application, wind and solar power generation, application of artificial intelligence to power systems, and software development.

Renke Huang (Member, IEEE) received the bachelor's degree in electric power system and automation from Shanghai Jiao Tong University, China, in 2006, the M.S. and Ph.D. degrees in E.C.E. from the Georgia Institute of Technology in 2009 and 2015, respectively. He is currently working as a Senior Research Engineer at Pacific Northwest National Laboratory, Richland, WA, USA. His research interests include power systems modeling and simulation, and application of advanced computing and machine learning technologies in power systems. 